\title{
Packet Loss Visibility for Higher Resolution Video on Portable Devices
}

${ }^{1}$ Mohammed Alreshoodi, ${ }^{2}$ Femi Adeyemi-Ejeye, ${ }^{2}$ Laith Aljobouri, ${ }^{2}$ Martin Fleury, ${ }^{3}$ Bander Alzahrani

${ }^{1}$ Qassim University, Buraydah, Saudi Arabia, ${ }^{2}$ University of Essex, Colchester, Essex, U.K,

${ }^{3}$ King Abdulaziz University, Jeddah, Saudi Arabia.

mo.alreshoodi@qu.edu.sa; \{eadeye,lamoha,fleum\}@essex.ac.uk; baalzahrani@kau.edu.sa.

\begin{abstract}
After adjusting for coding gain between the H.264 and HEVC codecs, a comparison is made between the two codecs' robustness to packet loss. A counter-intuitive finding arises that the less efficient codec is less affected by packet loss than the more efficient codec, even at very low levels of packet loss. The findings will be of interest to those designing portable devices that can display up to $4 \mathrm{kUHD}$ video.
\end{abstract}

\section{INTRODUCTION}

Ultra High Definition (UHD) broadcast transmission to portable devices was demonstrated at the 2014 Broadcast Asia conference employing Digital Video Broadcast terrestrial second-generation (DVB-T2) transmission. Compression was through a High Efficiency Video Coding (HEVC) standard codec. HEVC achieves up to $50 \%$ bitrate savings over the prior H.264/Advanced Video Coding (AVC) standard, because it was designed with higher resolutions in mind. However, as this paper details, there also may be a cost from greater compression efficiency in terms of exposure to packet loss, which can have an impact on quality even at low rates. In commercial terms, there is also a risk if a service to portable devices is not appreciated by its audience. Thus, this paper demonstrates counter-intuitive results in the sense that employing a more efficient codec, HEVC, results in a lower video quality than a less efficient codec, H.264/AVC, once packet loss is taken into account and coding gain is accounted for.

User expectation of mobile displays extends up to broadcast quality, owing to both the perceived sophistication of tablets and smart 'phones and the ability to off-load high-intensity computation to cloud processing. Despite those expectations, the reality is that video is still delivered to such devices over error-prone wireless networks. It is true that TCP-based pseudo-streaming with some form of HTTP Adaptive Streaming (HAS) can be applied to wireless communication. When an error occurs packets are retransmitted. However, though there are no errors present at the receiver, large buffers may be required, implying start-up delay affecting short video clips, and, for longer video sequences, there is a risk of increased and annoying stalls, along with quality fluctuations [1]. The impact of jitter is particular noticeable in interactive services such as mobile: video gaming, teleconferencing, and telemedicine.

However, if an Internet Protocol (IP)/User Datagram Protocol (UDP)/Real-time Transport Protocol (RTP) variety of streaming is employed then packet loss can directly impact upon the video quality. Most evaluations of video quality have been concerned with video resolutions below or occasionally up to HD and not beyond that. For example, Nightingale et al [2], analyzed network impairment on HEVC encoded video streams below HD resolution. Nevertheless, there is indirect evidence that changes in coding format result in different responses to packet loss. Thus, [3] showed that H.264/AVC quality drops dramatically for even low packet loss rates $(0.02 \%)$, while MPEG-2 quality drops by much less. The implication is that for equivalent transmitter HD quality the older codec achieves a better receiver quality once packet loss is taken into account. In this paper, we follow that implication [3] by investigating whether the same may apply to H.264/AVC and HEVC codecs at higher spatial resolution.

\section{Methodology}

To judge the relationship between Packet Loss Visibility (PLV) and spatial resolution, a streaming experiment was performed so as to judge the effect of packet scheduling at the point in time when packet losses occurred. The experiments involved 4kUHD along with Standard Definition (SD) and High Definition (HD) video. H.264/AVC and HEVC encoding was through the well-known FFmpeg implementations. Packet framing was by means of MPEG2-Transport Stream (TS) prior to UDP packetization. The packet sizes were close to the Ethernet maximum of about $1.5 \mathrm{kB}$. Error concealment at the decoder avoided complicating the interpretation by using basic previous frame replacement.

For the experiments, the choice of source test sequence was constrained by the availability of $4 \mathrm{k}$ versions. Thus, in most cases $4 \mathrm{k}$ adaptations of well-known test sequences were employed. Table 1 records the characteristics of the test sequences in terms of recommendation ITU-T P.910's Spatial Index (SI) and Temporal Index (TI).

In terms of choice of transmission parameters, choice of the lowest Constant Bitrate (CBR) for $4 \mathrm{kUHD}$ video was guided by the approximate savings of $35.4 \%$ of an HEVC codec over an H.264/AVC codec reported in [4]. Further, inhouse testing indicated that $4 \mathrm{kUHD}$ transmission is possible over recent wireless links (IEEE 802.11n and IEEE 802.11ad), because H.264/AVC can compress to $20 \mathrm{Mbps}$ if average bitrate rate control is utilized. Thus, a rate of around 13.5 Mbps for 4kUHD with an HEVC codec is arrived at, when an additional anticipated $0.1 \%$ PLR is anticipated. 


\begin{tabular}{lccl}
\multicolumn{4}{c}{ TABLE ITEST VIDEO SEQUENCES CONTENT TYPE } \\
\hline $\begin{array}{l}\text { Video } \\
\text { sequence }\end{array}$ & SI & TI & Motion classification \\
\hline Coast & 10.84 & 16.92 & Moderate \\
News & 17.52 & 21.24 & Moderate \\
Foreman & 19.71 & 38.29 & High \\
Sintel & 16.39 & 72.26 & High \\
\hline
\end{tabular}

\begin{tabular}{|l|l|l|}
\hline TABLE II CODEC PARAMETERS FOR TESTS \\
\hline Parameter & H.264/AVC & HEVC \\
\hline Profile & High $(5.1)$ & Main \\
\hline Processing unit & Macroblock $(\mathrm{MB})$ & $\begin{array}{l}\text { Coding Tree } \\
\text { Block }(\mathrm{CTB})\end{array}$ \\
\hline Processing unit size & $16 \times 16$ & $64 \times 64$ \\
\hline GoP size & 40 & 25 \\
\hline GoP frame structure & IPPPP... & IBBPBBP... \\
\hline CBR bitrates & $20 \mathrm{Mbps}$ & $13.5 \mathrm{Mbps}$ \\
\hline
\end{tabular}

\section{FINDINGS}

To calibrate the tests with packet loss, the error free results are first reported in Fig. 1. The findings suggest that HEVC produces marginally better quality video at a lower bitrate. In addition, higher resolution video results in lower objective Mean Opinion Score (MOS) ratings and lower motion videos have higher qualities. (Objective MOS was based on the Video Quality Metric (VQM) which has a high 0.9 correlation with subjective MOS [5].) However, the datarate was fixed whatever the resolution. This results in less compression for lower resolutions, as the Quantization Parameter (QP) varies to match the available bitrate. Moreover, the encoder's have been able to take advantage of the additional bitrate to improve the quality of low-motion videos. In other words, both codecs avoid simply increasing the bitrate artificially, by, for example, including more intra-coded MBs or CTBs.

Fig. 2 reports random packet loss of $0.1 \%$ for a selection of the video sequences. The H.264/AVC codec now (compared to Fig. 1) appears more resilient to packet loss than HEVC, resulting in higher quality ratings for H.264/AVC encoding. This finding strongly suggests that HEVC's more efficient encoding makes the video output more sensitive to packet loss, once the relative coding gains have been allowed for by scaling the CBR bitrates. Packet loss now serves to exaggerate the difference in quality already starting to show in Fig. 1 between lower (higher quality) and higher resolution (lower quality). Similarly, when packet losses occur, even at a low loss rate of $0.1 \%$, higher motion sequences such as Sintel suffer in quality much more than lower motion sequences such as Coast. Again a possible explanation, when a fixed rate CBR is involved, is that lower spatial resolution and lower motion video sequences will tend to be less compressed. Consequently, with more coded content per packet for lower resolution and lower motion videos, error concealment is better able to reconstruct missing packets. In addition, at higher QPs (lower quality), packet loss has more of an impact as the effect of temporal error propagation and the difficulty in performing error concealments are compounded.

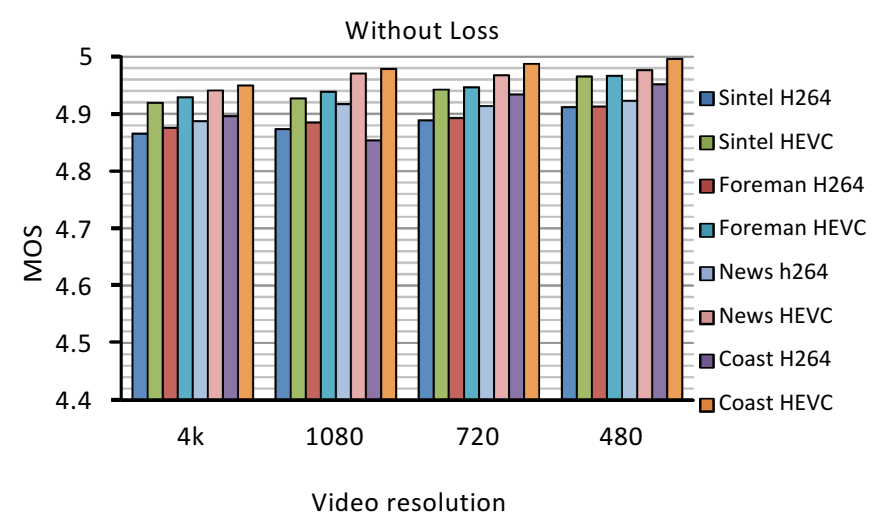

Fig. 1. Objective MOS video quality assessment for a range of resolutions with PLR $=0$ and either H.264/AVC or HEVC codec, showing improved MOS for HEVC when there are no packet losses

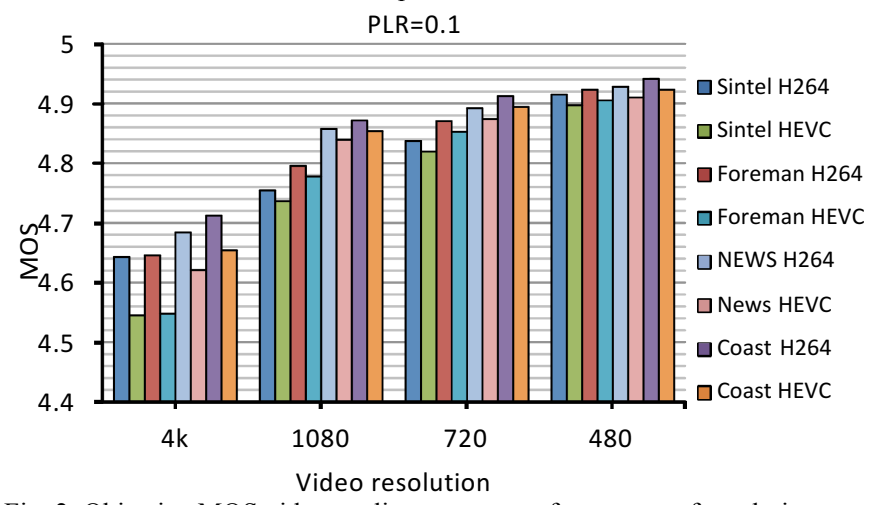

Fig. 2. Objective MOS video quality assessment for a range of resolutions with PLR $=0.1 \%$ and either H.264/AVC or HEVC codec, showing improved MOS for the older codec even when there are limited packet losses

\section{CONCLUSIONS}

Recent sports cameras capable of capturing 4kUHD video continue to use H.264/AVC, perhaps because emerging HEVC chipsets come with an unacceptable energy budget for their purpose. This paper's main finding is that the shelf-life of the H.264/AVC codec may be extended further whenever packet loss is a threat and interactive applications are considered.

\section{REFERENCES}

[1] M Seufert, S. Egger, H. Slanina, T. Zinner,T. Hoßfeld, and P. Tran-Gia, "A survey on Quality of Experience of HTTP adaptive streaming," IEEE Commun. Surveys \& Tutorials, vol. 17, no. 1, pp. 469-492, 2015.

[2] J. Nightingale, Q. Wang, and C. Grecos, "HEVStream: a framework for streaming and evaluation of high efficiency video coding (HEVC) content in loss-prone networks," IEEE Trans. Consumer Electron., vol. 58, no. 2, pp. 404-412, 2012.

[3] M.H. Pinson, S. Wolf, and G. Cermak, "HDTV subjective quality of H.264 vs. MPEG-2, with and without packet loss," IEEE Trans. Broadcast., vol. 56, no. 1, pp. 86-91, 2010.

[4] J. Ohm, G. Sullivan, H. Schwarz, T. Tan, and T. Wiegand, "Comparison of the coding efficiency of video coding standards including High Efficiency Video Coding (HEVC)," IEEE Trans. Circuits Syst. Video Technol., vol. 22, no. 12, pp. 1669-1684, 2012.

[5] M. Pinson, and S. Wolf, "A new standardized method for objectively measuring video quality," IEEE Trans. Broadcast., vol. 50, no. 3, pp. 312-322, 2004. 